\title{
ENHANCEMENT OF DAMAGE DETECTABILITY IN AIRCRAFT STRUCTURES USING THE FUSION OF NDT RESULTS
}

\author{
Marcin Zapłotny ${ }^{1}$, Andrzej Katunin ${ }^{1 *}$, Krzysztof Dragan ${ }^{2}$ \\ ${ }^{1}$ Institute of Fundamentals of Machinery Design, Silesian University \\ of Technology, Konarskiego 18A, 44-100 Gliwice, Poland \\ ${ }^{2}$ Air Force Institute of Technology, Ks. Bolesława 6, 01-494 Warsaw, \\ Poland
}

\begin{abstract}
Following the damage tolerance philosophy in aircraft design and operation, one of the most significant stages of maintenance is non-destructive testing of structures. It is, therefore, essential to use testing methods sensitive to particular damage types occurring in aircraft structures during operation. In this paper, the authors present a study on selection and comparison of methods of information fusion applied to testing the results of inspection of composite structures used in aircraft elements, obtained using various ultrasonic methods. The presented approach of fusion of ultrasonic scans allows for enhancement of damage detection and identification due to the presence of different parts of information about detected damage obtained from different initial information sources in a single resulting set. Such an approach can be helpful at the decision-making stage during inspection of aircraft elements and structures. Besides the methodology, the GUI-based software for performing fusion of various types of ultrasonic data is presented.
\end{abstract}

Keywords: aircraft composite structures, ultrasonic testing, information fusion, non-destructive testing.

\section{INTRODUCTION}

The aircraft industry, especially its military domain, has always been involved in developing and using both newest engineering solutions and materials. There is no difference in case of composite materials. Increasing share of composite components in the new aircraft is due to the weight-saving possibilities 
they introduce maintaining high durability. Composite structures, such as glass (GFRP) or carbon fibre-reinforced polymeric (CFRP) laminates present great specific strength and both corrosive and fatigue resistance.

Composites used in aviation, while creating new opportunities are also more demanding in terms of manufacturing and maintenance. The failure modes and flaws that can occur within their volume are different than those occurring in their metallic counterparts. Damage types of metallic structures are well known because of long history of studies over metals and their homogeneity on the macro-scale level. In contrast, the internal structure of composites is more complex and the damage and defects of composite structures can occur in many more other ways with respect to metallic structures.

For metallic and composite structures, various Non-Destructive Testing and Evaluation (NDT/E) methods are used. A lot of them are modifications of methods that were developed in the past for the testing of metallic elements. Their basic task is to give the possibility of monitoring the structure of the material without breaking its integrity.

Among the variety of available NDT/E methods, a group of ultrasonic testing (UT) methods are primarily used in aircraft inspection tasks due to their sensitivity and accuracy. These methods have become a standard in the testing of aircraft structures. UT can be performed manually or integrated into a system for automatic scanning. If the working transducer is moved in both planar axes, while its readings at each point are being saved and processed by the external computing unit, we have the possibility of creating C-Scan maps. These maps are a great tool for flaw detection and evaluation. Boeing ${ }^{\circledR}$ MAUS $^{\circledR}$ $\mathrm{V}$ is one of the most, if not the most versatile system existing on the market for that kind of application. It offers the possibility of mounting various types of transducers, not only for UT, and gives the tester map-like readings of the installed sensor set. Since Boeing ${ }^{\circledR}$ MAUS ${ }^{\circledR} \mathrm{V}$ can give us results of various types of UT tests and even methods based on eddy currents or mechanical impedance, it is possible to combine them to detect even smaller defects, in shapes sometimes not comfortable for some methods. This kind of approach to the results evaluation is referred to a information integration or, in other words, information fusion.

The aim of this study is to examine the potential for the enhancement of damage detection and identification in aircraft composite structures by applying various fusion algorithms to UT results acquired using different techniques. This work includes an overview of applicable fusion methods, examples of application of these methods based on UT results of real composite structures as well as presentation of the software developed for this purpose. 


\section{OVERVIEW OF FUSION METHODS}

In general, any process that combines information from at least two images into a single summary, can be called image fusion. The objective of image fusion is similar to the objective to data fusion in general, it is to reduce uncertainty and increase information carried by the images. In this branch of fusion, data appear in form of number arrays that represent features like colour, brightness, temperature, distance and some other properties of the scene. With the same input images, different fused image can be obtained, depending on what is considered the most important information that one wants to obtain. The main goal of image fusion should be to provide more information than can be given by an individual image [1].

Well applied image fusion should be the tool which allow us to overcome the deficiencies of individual images and deliver many valuable features of input images on one. Differences between images as listed below should be the reason to perform image fusion [2]:

- some features appearing in one but not in the other images,

- various objects and regions appearing in all or a few images but in different or even opposite contrast,

- when big disparities are present between different images, for example when images come from different sensors with different ranges, resolution or principle of work.

Even though image fusion itself is not that young branch of analysis, a number of scientific papers published related to it has rapidly increased over last couple of years. New methods of performing various actions related to image fusion as well as improving existing is taking place in the moment of writing this work [3]. Image fusing algorithms can be classified into three categories: low, medium and high level algorithms. These categories for purpose of image fusion are called in order pixel-level, feature-level and decision-level fusion and are based on image representation level at which fusion takes place [4].

Pixel-level fusion of images has found wide application in medical imaging, computer vision and remote sensing. It is usually easier and more time effcient than its feature level counterpart. It also covers more of original information. As a drawback, its results are more prone to registration errors and noise. Basic image fusion would combine each color band of a picture with some kind of fusion rule, for example taking average value between each pixel colour band value of images. This creates a new image with every pixel having average RGB values of original images. Although it is very simple to perform, it greatly reduces contrast of the image and does not permit much more than just blending several pictures into one. Because of that various image decomposition methods 
were developed to describe images in a way that allows for more sophisticated pixel-level fusion. As a result, pixel-level image fusion methods consist of three main stages [3]:

- image transform, which is often connected with image decomposition,

- fusion of the obtained and selected coeffcients,

- inversion of the transform, preferably without increasing the noise and level of artifacts present in the original images.

Multi-scale decomposition is a long-established tool for fusion of images as well as different image processing applications. As a general rule for pixellevel image fusion, multi-scale decomposition transform is used to obtain a representation of an image in which features of the images are represented in a space-frequency domain. Then, the fusing of those multi-scale representations is performed to obtain a fused multi-scale representation according to specific fusion rule. At the end, inversion of decomposition is enrolled to go back to the original representation of the fused image. In this method, there are two choices: choice of a decomposition method and the choice of a fusing strategy.

One of such techniques is a very useful technique of pyramidal image coding based on Gaussian and Laplacian pyramids described by the authors of [5]. It allows for pyramidal decomposition of images for the purpose of their storage, compression and fusion. Many decomposition techniques based on this approach developed more recently, i.e. wavelet, curvelet or contourlet, etc. One can consider the pyramid representation to be the predecessor of multiresolution analysis and scale-space representation even though it is functional as it is.

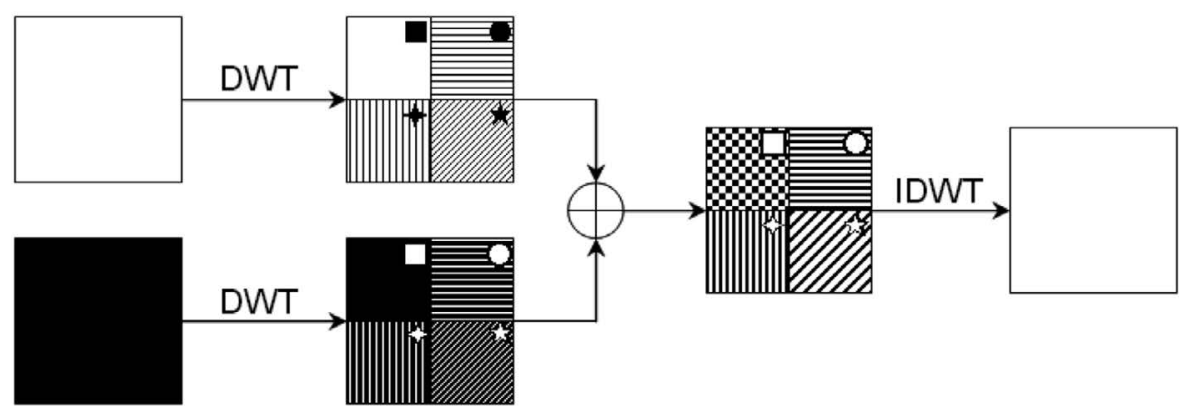

Fig. 1. General scheme of wavelet-based fusion [7].

The above-mentioned wavelet-based approach seems to be a promising tool for data fusion. The most important step in its algorithm is the formation of the fusion pyramid and deciding on a fusion principle. Different selection rules for sub-images fusion can be implemented leading to different results. For example, 
maximum, average and weighted average criteria can be applied as fusion criteria. Also, image decomposed with discrete wavelet transform (DWT) can be fused with other types of image decompositions, like the IntensityHue-Saturation (IHS) method or Panchromatic (PAN) images, making DWT decomposition one of the most popular method used in present image fusion [6]. The general scheme of the wavelet-based fusion, which uses DWT and its inversion, IDWT, is presented in Fig. 1.

Another approach is the sparse coding, which simulates the mechanism of human vision. The word sparse in this case refers to the fact that only few non-zero elements can effciently enough represent the original image's saliency information. By exploitation of the characteristic of sparse coeffcients used to describe the given signal, they can be used for image fusion. In theSparse Representation (SR) system, the signal is described by sparse linear combination of atoms that are selected from a dictionary of prototype signal atoms. On contrary to wavelet transform, fused image is not globally created, making changes in coeffcients without influencing the whole picture but only corresponding patch. Two main characteristics of SR are its sparsity and overcompleteness. By these terms, it can be understood that the number of basic atoms in the dictionary exceeds the image pixels and that only a few descriptions are needed to describe the structure of the corresponding object. Sliding window technique is usually used to better capture local salient features and to keep shift invariance. This method has been successfully used in applications such as compression, denoising, feature extraction and feature classification [8]. A basic fusion scheme relying on this approach is presented in Fig. 2.

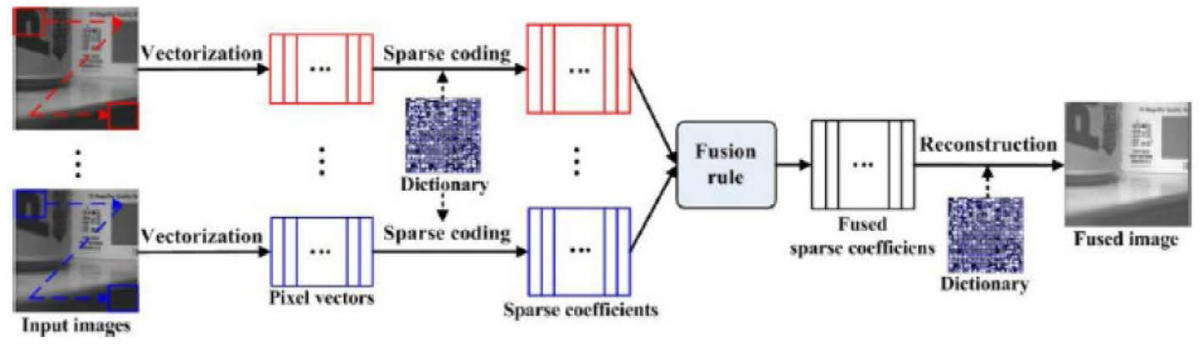

Fig. 2. Schematic diagram of generic SR based image fusion method [3].

In modern technology, for colour image storing, the Red-Green-Blue (RGB) system is widely used. Each pixel stores information about the intensity of three basic colours which are than mixed by a graphic device. The image is treated as a set of three separate matrices for each base colour. Because each pixel has three numerical coordinates, it can be visualized as a cube model of colour 
representation. The RGB system has found wide use as modern television and computer screens that use this technology with a great success. It is though not a good way to store image data for fusion because it introduces problems with contrast and feature recognition.

The IHS is the system of storing colourful image data in a different way. It is based on two identical cones, connected at their bases, instead of the cube. It is sometimes confused with HSV (hue, saturation, value) or HLS (hue, luminance, saturation), which are slightly different not only in swapping intensity for value and luminance respectively but also in saturation and hue definitions. In some literature, the letters are swapped, forming HIS, which does not change anything in the concept. The goal of IHS is to facilitate separation of shapes in the image [9].

Definitions of IHS components are as follows [10]:

- Intensity - Brightness relative to the brightness of a similarly illuminated white. It ranges between $[0,1]$, where ' 0 ' means total black, and ' 1 ' highest obtainable white.

- Hue - Colour itself in the form of an angle between $\left[0,360^{\circ}\right]$. $0^{\circ}$ corresponds to red, $120^{\circ}$ to green and $240^{\circ}$ to blue.

- Saturation - Colourfulness of a stimulus relative to its own brightness. It varies between $[0,1]$, where ' 0 ' means totally weathered colour, and ' 1 ' its full colourfulness.

Hue is the most meaningful when saturation is close to ' 1 ', and least meaningful when Intensity approaches either of its boundary values.

The IHS fusion technique is very popular in remote sensing community. It is widely used in fusing of high-spacial, low-spectral PAN images and Multispectral (MS) colour images with low-spacial and high spectral resolution for obtaining both high-spectral and high-spacial images. It is often used when fast computing of the fused images is necessary. In that kind of application the Intensity component of the MS image is usually replaced with PAN histogram, after their size match [11]. It is also possible to fuse PAN images decomposed using the wavelet transform with MS images transformed into IHS colour space $[11,12]$.

Out of many approaches to image fusion developed over years, the authors have chosen two for application to the C-scan UT results:

- Wavelet-based image fusion, due to its widespread applicability to fusion problems. The wavelet-based approach allows for setting many parameters, beginning with a vast amount of wavelets types and decomposition levels, ending with many combinations of fusion rules for approximation and detail coeffcients matrices. 
- Image fusion connected with conversion of images into different encoding systems, which has found many applications in fusion of live or very big images. In this case, it is worth evaluating if the fusion method that has found such wide application in satellite and medical image processing can be also used with success for the UT results fusion. Results of UT being provided in multiband and PAN images is the main reason to choose methods connected with that kind of fusion.

\section{APPLICATION OF FUSION ALGORITHMS FOR UT DATA PROCESSING}

\subsection{Experimental data}

Data used for analyzing algorithms is in the form of images resulting from the C-Scan UT of composite structures. The testing rig was formed by the Boeing ${ }^{\circledR}$ MAUS $^{\circledR} \mathrm{V}$ system. It is worth to mention that images in C-Scan mode are representations of measurements, not measurements as such. They allow for much easier reading of results. Originally, the results come as a number matrices, while in post-processing they are changed into a graphical form. The results of UT performed by the Boeing ${ }^{\circledR}$ MAUS $^{\circledR} \mathrm{V}$ test rig are presented in Fig. 3. The scales are parts of image processing and allow to connect colours (Time of Flight - depth mode) and brightness (amplitude - attenuation mode) with mapped results. Three purposely-introduced delamination areas are present in the tested structure.
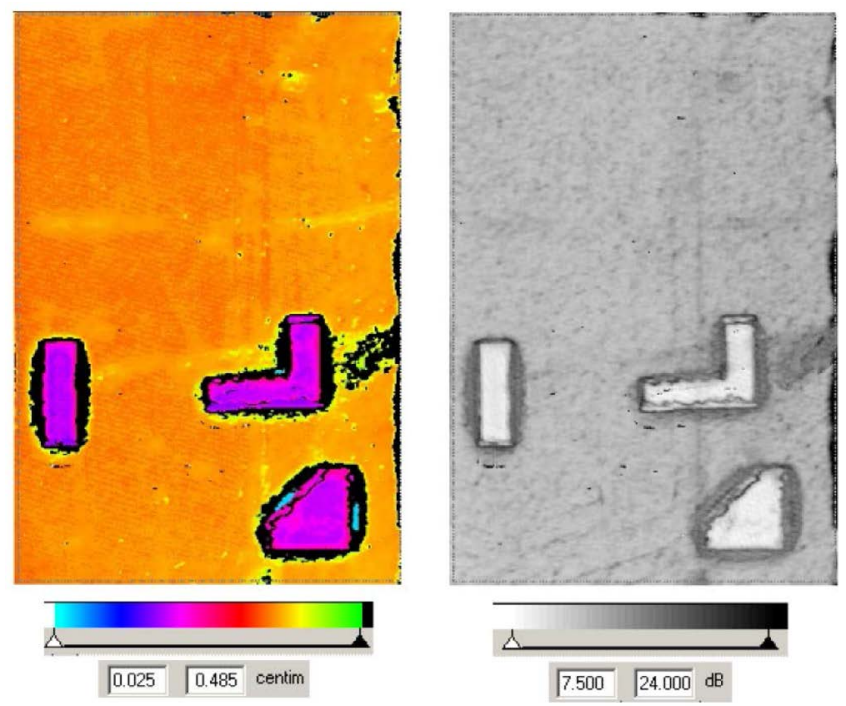

Fig. 3. Exemplary results obtained from UT scanning. 
The first image is a colour-map in which each pixel represents a single point's depth measurement. It is the main source of information about the flaw because one can clearly distinguish different depths of flaws present in the structure. In the dedicated software provided by the system manufacturer, one can adjust the colour spectrum to the values present in the matrix.

The second one is usually a panchromatic map of ultrasound wave attenuation at each point of the measured area. In the attenuation map presented, white colour is related to point of the lowest registered wave amplitude, while black shows the highest value of it. This result can tell us more about the flaw characteristic seen in the depth measurement representation and to filter out errors that can be introduced by, for example, too rough surface of the specimen.

For fusion purposes, both images should have the same resolutions and be coregistered to each other. It is not a problem in this case since all measurements are enrolled during one procedure and cover exactly the same area. It happens to be a problem, when fusion of images from different resolution sources is to be performed or when sensors make measurements at different locations.

\subsection{Fusion principle}

The general procedure for obtaining the fused image is visualized in the scheme presented in Fig. 4. The resulting image should provide information given by both input images without increasing unwanted properties, such as noise, or changing colour spectrum present in the first image. Additionally, any improvement to defect distinguishability or a sign of artifact elimination is desirable.

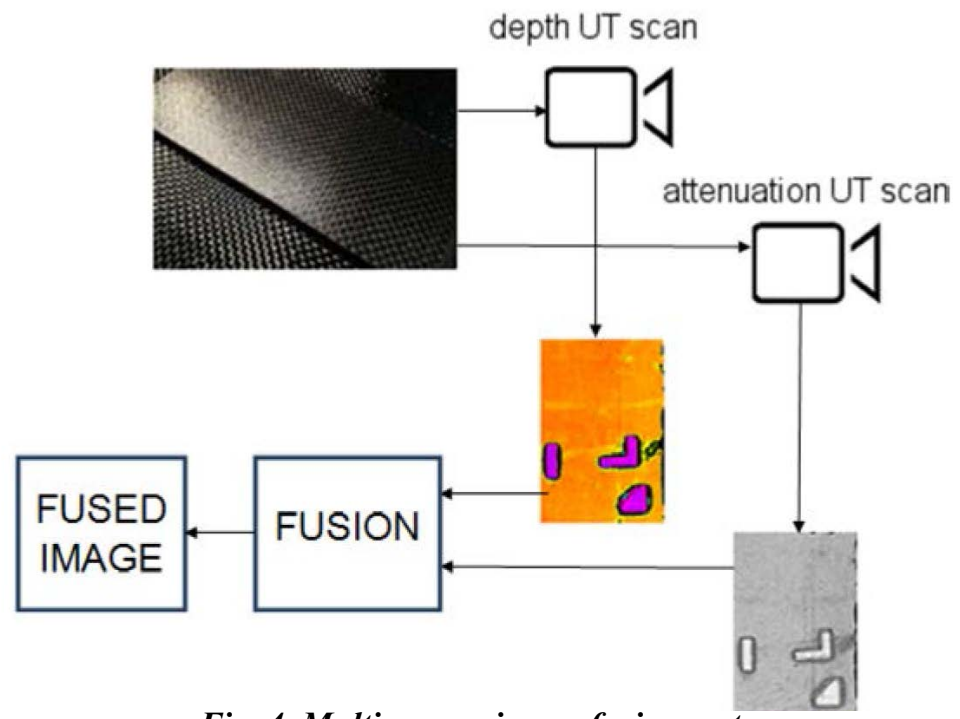

Fig. 4. Multi-sensor image fusion system. 
The first algorithm used the wavelet-based fusion, which uses MATLAB ${ }^{\mathrm{TM}}$ Image Toolbox ${ }^{\mathrm{TM}}$ and Wavelet Toolbox ${ }^{\mathrm{TM}}$. It performs 2-D DWT on both images, fuse their coeffcients using the defined rules (Coeffcients-fusion) and carry 2-D IDWT to obtain a fused image (see Fig.1). The changeable parameters of the algorithm are:

- family and order of the fusing wavelet. The choice consists of three types of orthogonal wavelets differing in symmetry and compactness, commonly used in image and text processing. Particular wavelet families were chosen to present a representative sample of three, that has both similarities and differences among them:

- Daubechies (orders from 1 to 10),

- Symlets (orders from 2 to 8), least symmetric, very compactly supported,

- Coiflets (orders from 1 to 5), more symmetric then previous, bigger support size.

- Number of decomposition levels (from 1 to 10).

- Rules for fusing of approximations and details matrices respectively:

- min, max, mean, img1, img2, rand.

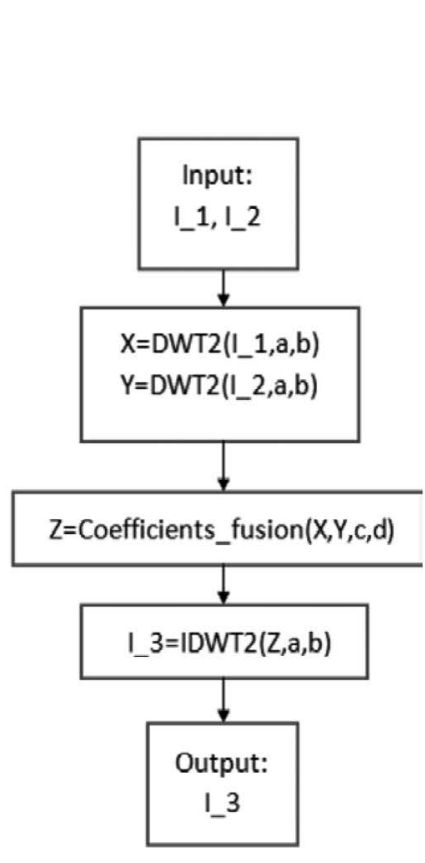

a)

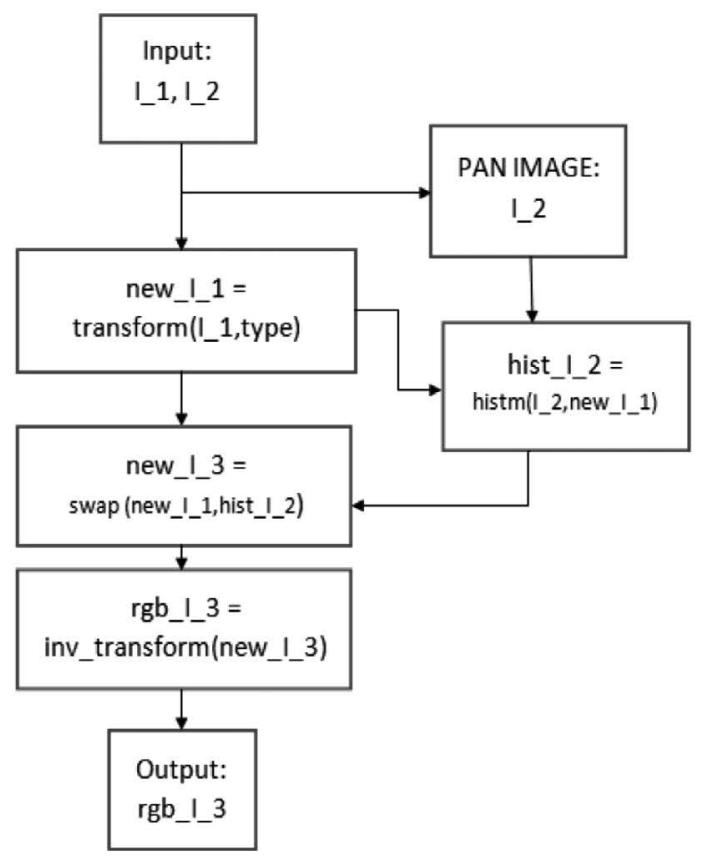

b)

Fig. 5. Data flow schemes for a) wavelet-based and b) encoding transform-based fusion algorithms. 
The resulting scheme is presented in Fig. 5a.

The other approach was based on different encoding transform-based image fusion algorithms. The task of this algorithm is to change encoding of the multispectral image (depth measurement) originally stored in the RGB system, to a type in which one of the bands can be replaced with a PAN image (attenuation measurement), which is histogram-matched (histm) to this particular band. This algorithm should preserve colours of the first image while changing their intensities to correspond with an attenuation measurement image. Three different encoding systems can be used as new ones for the multispectral image: IHS, YIQ and HLS. Additionally, the transform to IHS system can be performed in various ways, giving slightly different results. The details on the pickable transforms and their inversions can be found in [6]. The scheme for this type of algorithms is presented in Fig. 5b.

\section{RESULTS OF FUSION OF UT SCANS}

In order to visualize differences between the considered algorithms and their applicability, various fusion results corresponding to them were presented. The results were analyzed not only from the damage identification perspective, but also in terms of how they can lead to further post-processing of C-scans. Fusion was performed on image pair presented in Fig. 6 .
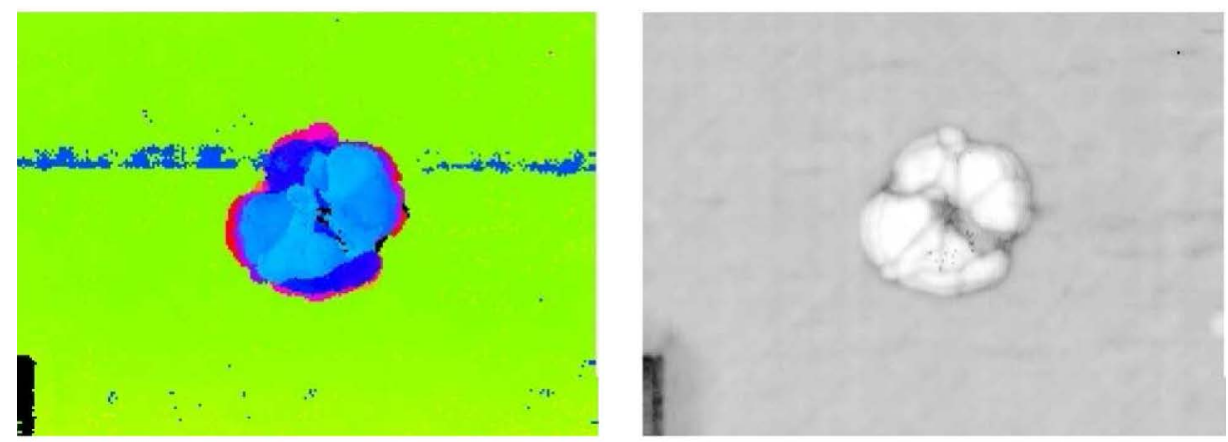

Fig. 6. Selected scans of a composite structure with barely visible impact damage: input depth measurement multiscale image a) and attenuation measurement PAN image b).

The wavelet-based fusion algorithm has a vast number of possible permutations according to the available tools in MATLAB ${ }^{\circledR}$ Wavelet Toolbox ${ }^{\mathrm{TM}}$ (exactly 7920) and because of it only selected results have been showed. They are grouped in three basic categories, in which only one fusion parameter changes between them. This allows to show how this parameter influences the results of the fusion. A common feature of images created in wavelet-based 
fusion is distortion. Different sets of results are presented in Figs.7-9. Additionally, various versions of encoding transform-based algorithms were applied to the considered pair of the UT scans (Fig. 6) in order to test their effectiveness. The results are presented in Fig. 10.

a)

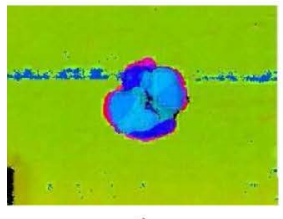

c)

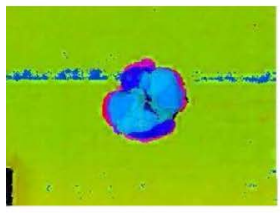

e)

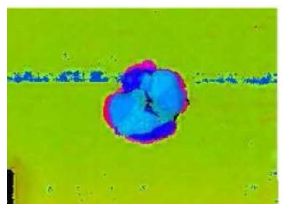

Fig. 7. Fusion done to decompositions at 2nd level with fusion parameters 'min' and 'max' for approximations and details, respectively. Wavelet used are: a) db1 b) db6 c) sym 2 d) sym8 e) coif1

$$
\text { f) coif5. }
$$

a)

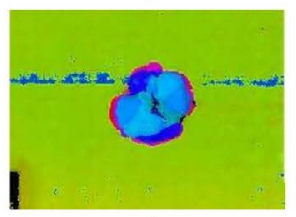

c)

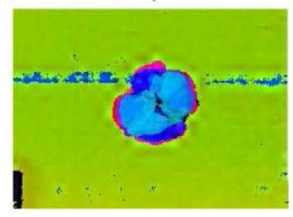

e)

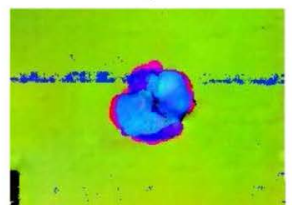

Fig. 8. Images fused using symlet of 4th order with fusion parameters 'min' and 'max', at different decomposition levels. a) $1^{\text {st }}$, b) $2^{\text {nd }}$, c) $3^{\text {rd }}$, d) $5^{\text {th }}$, e) $7^{\text {th }}$, f) $10^{\text {th }}$.

In Fig. 7, there difference is difficulty observable. The only change worth mentioning is associated with the differences in region of lighter background pixels around the main flaw. For the Haar wavelet (also db1), the region is a lot more pixelated. Also, for higher wavelet orders, flaw edges tend to be more distorted.

In Fig. 8, two changes in patterns can be noticed without objective evaluation of the results:

- change in the region around the main damage in the centre of the picture. With a higher "level" parameter, the region of higher brightness without details is getting bigger, at $5^{\text {th }}$ level, shown on d) contrast is the highest;

- difference that can be noticed in the gradient of colours in the main flaw. For higher levels, colours change more to match attenuation measurement. 
a)

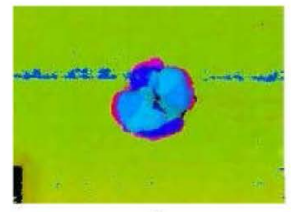

c)

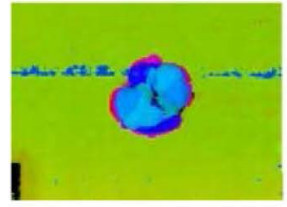

e)

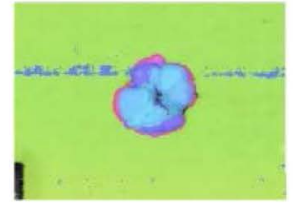

Fig. 9. Images fused using 4th order symlet at the $2^{\text {nd }}$ decomposition level.

Fusion rules are as follows: a) min, $\max , b) \max , \min , c) \min , \min , d) \max$, max, e) mean, mean, f) img2, max for, respectively, approximations and details. a)

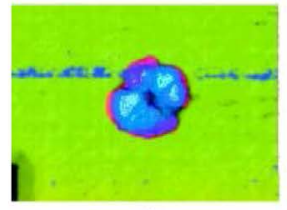

c)

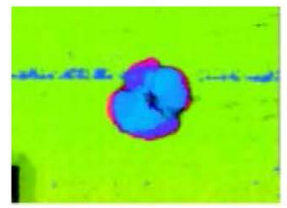

e)

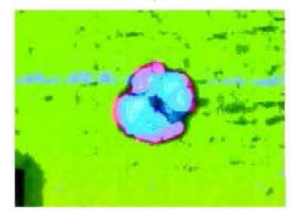

Fig. 10. Fusion based on a) IHS1,

b) IHS2, c) IHS3, d) IHS4, e) YIQ, f)

HLS colour system. b)

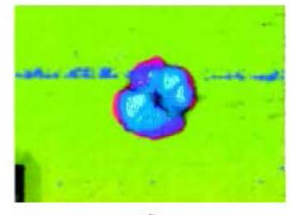

d)

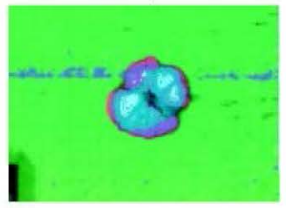

f)

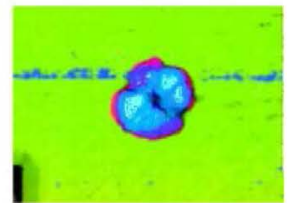

For the results presented in Fig. 9, max or min values of both parameters change colours to match the PAN image more $(b, d)$; in those pictures, edges are extracted better. Max value for the second parameter produces a sharper image, which is a desired result. In b), d) and f), the resulting images are more similar to the attenuation measurement image. The parameter sets used for fusion e) and f) give results which can be considered as fails.

In Fig. 10, all results besides d) have a higher colour preservation of the first input image than wavelet-based. IHS3 in c) smooths an image too much. a), d) and f) are similar in injection of the PAN image with a) having the highest level of details. The YIQ model shown in e) introduces too many artifacts. Based on these six images, subjectively, the IHS1 decomposition is head above the competition.

\section{EVALUATION OF EFFECTIVENESS OF FUSION ALGORITHMS}

In previous section it was mentioned that the fused images obtained with use of the considered algorithms, especially the wavelet transform, do not differ much. It is characteristic for the wavelet image fusion and such results were expected. Introducing number parameter that could describe fusion results helps 
in their quality evaluation. Objective fusion quality measurement is not an easy task, especially because the features of fused images under consideration are hardly convertible into mathematical expressions. The best evaluation should be performed by inspector who himself would decide which features of fused images are the most important and which algorithm settings suits obtaining them. However, some kind of objective measure, even if not perfect, could bring at least a little help into the decision-making process. In this section, four quality measures are formulated. The first, second and forth are taken from literature related to image fusion, while the third is a simple measure of calculating overall difference between two images.

All image matrices are normalized, which means that values are in the range of $[0,1]$ instead of $[0,255]$. Sadly, quality measures should not compare different algorithms or fusion of different image sets. It is due to the sensitivity to any changes between images. Additionally, $Q_{w}$ has initially lower values the more input images are different from each other. On the other hand, they provide additional insight when comparing subtle changes in result images in the range of the same input data, for example, when changing the wavelet type in waveletbased image fusion algorithm.

The first considered approach of an evaluation of fusion quality is the fusion quality index originated from [13] and successfully used in similar studies [7]. It is based on comparing all possible local windows (in this case of size $8 \times 8$ pixels) taken from all three images, and comparing the fused image (A) with the first (B) and second (C) initial images, separately:

$$
Q_{w}(A, B, C)=\frac{1}{|W|} \sum_{w \in W}\left(\lambda_{A}(w) Q_{0}(A, C)+\lambda_{B}(w) Q_{0}(B, C)\right)
$$

where $W$ is a family of all local windows $w$, which slides pixel-by-pixel over the entire image, and $|W|$ is the cardinality of $W, \lambda_{i}(w)$ is the local weight enclosed in the range of $0-1$, which have a form:

$$
\lambda_{A}(w)=\frac{s(A \mid w)}{s(A \mid w)+s(B \mid w)},
$$

$s(A \mid w)$ and $s(B \mid w)$ are the saliencies of images $A$ and $B$ in window $w$, which should reflect the relevance of a given image in $w$, and $Q_{0}(\cdot, \cdot)$ is the quality index following [14]:

$$
Q_{0}(A, B)=\frac{4 \sigma_{A B} \bar{A} \bar{B}}{\left(\bar{A}^{2}+\bar{B}^{2}\right)\left(\sigma_{A}^{2}+\sigma_{B}^{2}\right)},
$$


where $\bar{A}$ and $\bar{B}$ are the mean values of $A$ and $B$,

$$
\begin{gathered}
\sigma_{A}^{2}=\frac{1}{M N-1} \sum_{m=1}^{M} \sum_{n=1}^{N}(A(m, n)-\bar{A}) \\
\sigma_{A B}=\frac{1}{M N-1} \sum_{m=1}^{M} \sum_{n=1}^{N}(A(m, n)-\bar{A})(B(m, n)-\bar{B})
\end{gathered}
$$

$M, N$ are the sizes of the image and $m, n$ are the elements of it. Considering the sliding window approach (4) could be transformed to the local quality index, which takes the form:

$$
Q_{0}(A, B)=\frac{4 \sigma_{A B} \bar{A} \bar{B}}{\left(\bar{A}^{2}+\bar{B}^{2}\right)\left(\sigma_{A}^{2}+\sigma_{B}^{2}\right)},
$$

Next three parameters, namely, the signal to noise ratio (8), colour change (9), and deviation index (10):

$$
\begin{gathered}
S N R=\sqrt{\frac{\sum_{i}^{M} \sum_{j}^{N} \sum_{k}^{O} A(i, j, k)^{2}}{\sum_{i}^{M} \sum_{j}^{N} \sum_{k}^{O} A(i, j, k)-B(i, j, k)^{2}}} \\
C_{-} C=\frac{\sum_{i}^{M} \sum_{j}^{N} \sum_{k}^{O} \mid B(i, j, k)-A(i, j, k)}{M N O} \\
\frac{1}{M N O} \sum_{i}^{M} \sum_{j}^{N} \sum_{k}^{O} \frac{\mid B(i, j, k)-A(i, j, k)}{B(i, j, k)}
\end{gathered}
$$

are constructed specially for the purpose of providing measure of multispectral and PAN images. They focus on comparing original multispectral and resulting images. The PAN image which represents attenuation measurement is skipped. In the case of SNR, values can be in the range of $[0, \infty]$, where the higher value corresponds to better fusion, while in the other two cases, values can be in the range of $[0,1]$, where the lower value corresponds to lower colour change, thus, a better fusion result. The results obtained are stored in Table 1 for the waveletbased algorithm and in Table 2 for encoding transform-based algorithms. The results presented in Tables 1 and 2 correspond to the obtained fused images presented in Figs. 7-10. 
Table 1. Quantitative evaluation of fusion quality during application of the waveletbased fusion algorithm

\begin{tabular}{|c|c|c|c|c|}
\hline $\begin{array}{c}\text { Fusion parameters } \\
\text { (wavelet type, level, AFUS, } \\
\text { DFUS) }\end{array}$ & $\mathbf{Q}_{\text {w }}, \mathbf{1 1 0}^{-4}$ & $\mathbf{S N R}$ & $\mathbf{C C}$ & DI \\
\hline (db1, 2, min, max) & 2.25743 & 0.103816 & 0.067874 & 0.175564 \\
\hline (db6, 2, min, max) & 2.45331 & 0.103604 & 0.0685373 & 0.167211 \\
\hline (sym2, 2, min, max) & 2.52967 & 0.103672 & 0.0678265 & 0.163041 \\
\hline (sym8, 2, min, max) & 2.25029 & 0.103625 & 0.0679379 & 0.154521 \\
\hline (coif1, 2, min, max) & 2.221 & 0.103678 & 0.0679061 & 0.158128 \\
\hline (coif5, 2, min, max) & 2.24777 & 0.103618 & 0.0678855 & 0.154819 \\
\hline (sym4, 1, min, max) & 1.26667 & 0.103496 & 0.0668084 & 0.11405 \\
\hline (sym4, 2, min, max) & 2.29863 & 0.103634 & 0.0678181 & 0.154294 \\
\hline (sym4, 3, min, max) & 3.31306 & 0.103899 & 0.69008 & 0.213268 \\
\hline (sym4, 5, min, max) & 5.29322 & 0.104651 & 0.0697985 & 0.336835 \\
\hline (sym4, 7, min, max) & 31.0706 & 0.106248 & 0.0649802 & 0.566164 \\
\hline (sym4, 10, min, max) & 20.4526 & 0.10805 & 0.075236 & 0.958875 \\
\hline (sym4, 2, min, max) & 2.29863 & 0.103634 & 0.0678181 & 0.154294 \\
\hline (sym4, 2, max, min) & 9.25908 & 0.14789 & 0.341224 & 11.8615 \\
\hline (sym4, 2, min, min) & 0.65117 & 0.103154 & 0.0820325 & 0.30498 \\
\hline (sym4, 2, max, max) & 10.0605 & 0.141968 & 0.338063 & 11.7856 \\
\hline (sym4, 2, mean, mean) & 4.29472 & 0.120436 & 0.201053 & 5.95899 \\
\hline (sym4, 2, img2, max) & 10.0338 & 0.138844 & 0.398945 & 11.8489 \\
\hline
\end{tabular}

In the first section of the table, the influence of a wavelet type was evaluated based on the measure parameters. It can be seen that the values vary at 'max' by few percent for Deviation Index measure. For other measures, changes are negligible from the evaluation perspective. It can be concluded that for this dataset, objective measurements do not indicate much difference between wavelet families and types available for use in the application.

The situation looks much different for the second section of data for measurements, where the impact of decomposition levels was evaluated. For higher levels, fusion quality indicated by $Q_{w}$ rises, and the same concerns SNR. 
Fusion at higher decomposition levels provides better background smoothing. However, the DI measure result is the best for lower decomposition levels. The third section consists of images that strongly vary. Images with the fusion rules parameters ' $\max$, min', 'max, max' and 'img2, max', which are more similar to the panchromatic measure outcome, provide the best results in terms of first two measures. The rest of the images, which take more from a multiband image (depth measurement), exhibit better colour preservation and lower deviation.

Table 2. Quantitative evaluation of fusion quality during application of encoding transform-based fusion algorithms

\begin{tabular}{|c|c|c|c|c|}
\hline Fusion type & Qw, $\times \mathbf{1 0}-\mathbf{2}$ & SNR & CC & DI \\
\hline IHS1 & 3.14456 & 12.6914 & 0.453047 & 0.414178 \\
\hline IHS2 & 3.14456 & 15.1894 & 0.293773 & 0.390364 \\
\hline IHS3 & 3.19466 & 16.9748 & 0.0268889 & 0.294927 \\
\hline IHS4 & 2.72484 & 10.4115 & 0.180763 & 3.92323 \\
\hline YIQ & 0.067881 & 7.9287 & 0.058197 & 0.747677 \\
\hline HLS & 3.14456 & 15.1894 & 0.293773 & 0.390364 \\
\hline
\end{tabular}

In this case, evaluation results are much more clear and undoubted. The IHS3 transform gives the best results in terms of every measurement procedure. Compared to other types, IHS3 provides the best fusion quality with lowest noise and colour change. The main reason for such good results is that it does not put close-to-white areas inside of the main flaw area in the centre of the image. Fusion using YIQ encoding of the first image gives by far the worst results because of high colour change and artifacts appearance.

\section{SOFTWARE DEVELOPED FOR FUSION OF ULTRASONIC SCANS}

For the purpose of results presentation, the evaluation of algorithms and basic image organization, Graphical User Interfaces (GUIs) corresponding to each type of algorithm were developed. Because they were programmed in MATLAB $^{\circledR}$ environment, they are tightly intertwined with developed fusion algorithms. For running them smoothly, the user should have MATLAB ${ }^{\circledR}$ with Wavelet Toolbox ${ }^{\mathrm{TM}}$ and Image Processing Toolbox ${ }^{\mathrm{TM}}$ installed. This section contains a short description of both programs. The views of windows of these programs, namely, wavelet-based and encoding transform-based algorithms, are presented in Figs. 11 and 12, respectively. 


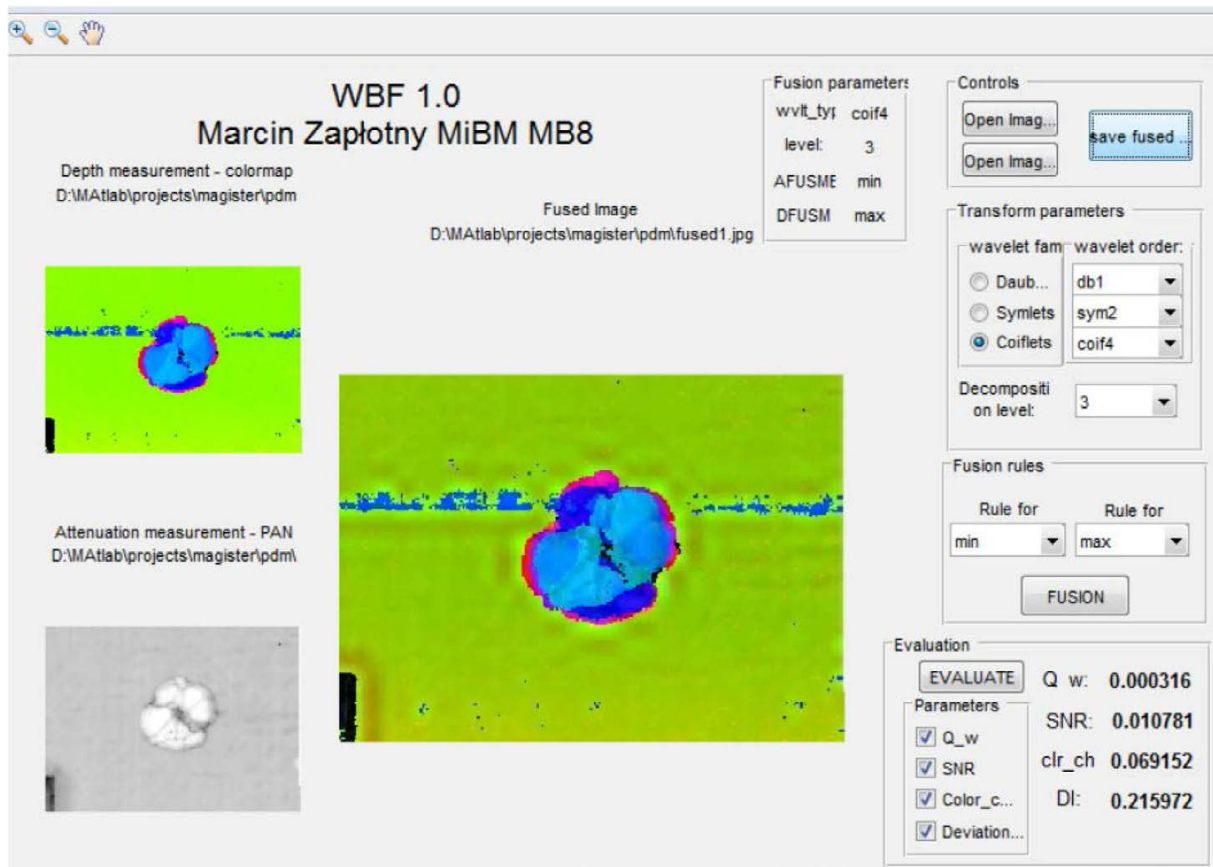

Fig. 11. Wavelet-based fusion program GUI layout [6].

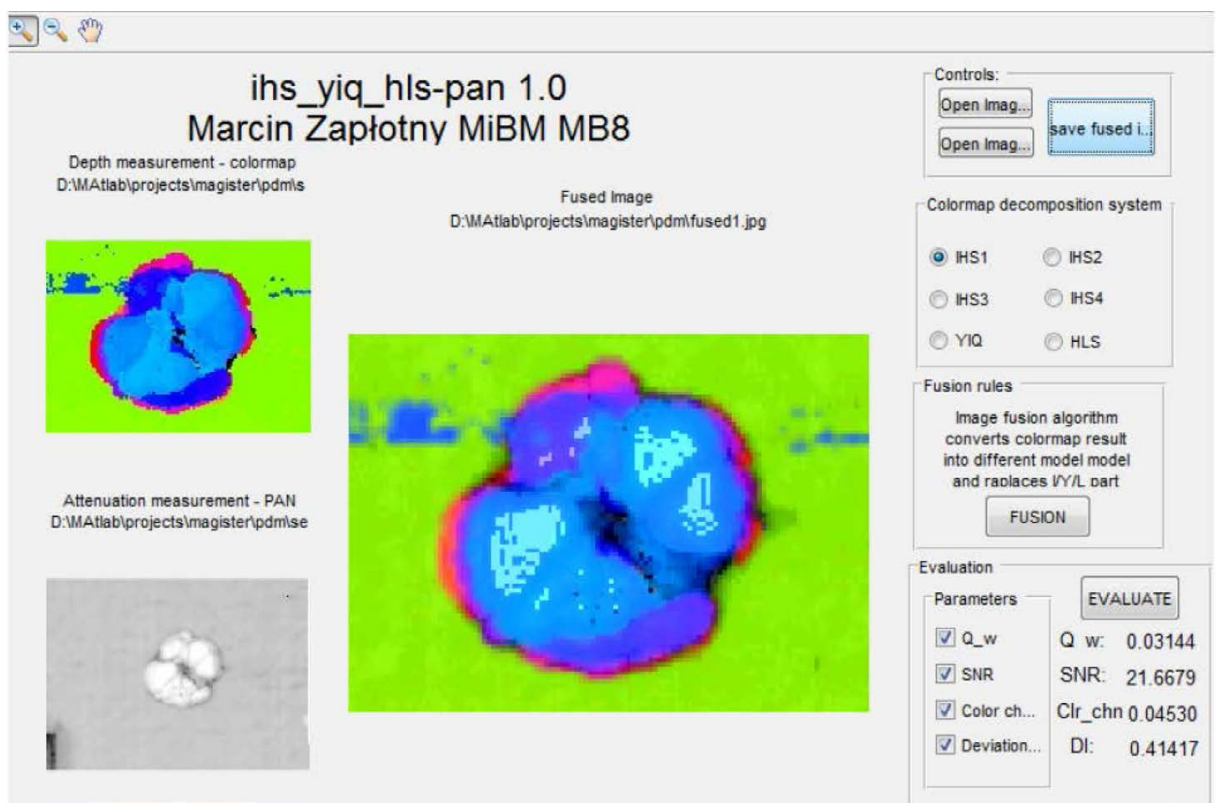

Fig. 12. Encoding transform-based fusion program GUI layout [6]. 
While using both programs, it is important to load images that have the same resolution. Since the purpose of these GUIs was to work with the considered C-Scan images of the same size, no functions for image resizing or image format conversion were used. It is also important to know that some functions of the programs perform a vast number of operations, so fusing high resolution images using the wavelet-based algorithm at decomposition levels higher than 3 can take a lot of time, even on fast PCs. The same applies to calculating $Q_{w}$ measure of high resolution fused results. If one wants to pick the right algorithm for C-Scan fusing for its needs, it is suggested to lower resolution of the loaded images, check high number of fusion parameters permutations and after choosing parameters wanted, perform full-time analysis of higher resolution images.

Both GUIs work in a "proportional mode" meaning they can adjust to user's resolution. Because of this feature, they should maintain all functionalities on every screen, although the experience and visuals of the programs can vary on different monitors.

\section{SUMMARY}

A problem related to any multi-sensor measurements is segmentation of obtained information. In the case of UT, some features of the tested structure can be seen in the results obtained by attenuation measurement, but cannot be seen the main depth measurement result.

After performing of theoretical research, two image fusion approaches were taken into further consideration. These are the wavelet transform-based image fusion and different encoding transform-based image fusion algorithms. Together with algorithms, Graphical User's Interfaces were developed for more intuitive fusion and the presentation of its results. After the development process, the algorithms for applying these fusion types were verified against experimental results in the form of C-Scans of damaged composite structures similar to those used in aircraft design.

The results, corresponding to different algorithm parameters, were compared with original images and with each other. As an outcome of this process, some initial conclusions could be formulated. They are as follows:

- algorithms are able to perform respective fusion schemes and either blending information provided by two different images (for the first algorithm) or injecting information carried by panchromatic attenuation measurement into a multiscale depth measurement image (for the second algorithm); 
- the main feature of the resulting images is the introduction of details into the background areas of images presenting depth measurement results;

- for particular parameters of the wavelet-based algorithm:

- better segmentation of the flaw area from the background was obtained,

- small artifacts were polished,

- image sharpening was achieved.

- for particular systems of different encoding transform-based image fusion:

- better colour preservation was achieved than for first algorithm,

- attenuation measurement information was injected into background and flaw ares of multiscale images.

After the verification of the algorithms, the authors decided on using objective measures for the evaluation of the results. Four measures were applied to selected results. Based on these results it is possible to formulate additional conclusions:

- wavelet family, picked out of three available does not make much difference in terms of fusion quality;

- higher decomposition levels for the first algorithm give more denoised image and provide a better flaw area segmentation from the background;

- for particular parameters of both algorithms, the results are completely off and don't give results aiding the damage evaluation;

- second algorithm is better at preserving colours, of all encoding types available for use, IHS3 gives the best results.

The obtained results of algorithms considered gave promising results, as it was found that using different fusion methods and parameters one can achieve enhancement of information provided by separate UT results, which can be useful for inspectors of aircraft composite elements during analysis and decisionmaking process when classifying the changes observed in C-Scans as flaws.

\section{REFERENCES}

[1] Goshtasby A.A., Nokolov S., Image fusion: Advances in the state of the art, Information Fusion, 8(2), 114-118, 2007.

[2] Piella G., A general framework for multiresolution image fusion: from pixels to regions, Information Fusion, 4(4), 259-280, 2003.

[3] Li S., Kang X., Fang L., Hu J., Yin H., Pixel-level image fusion: A survey of the state of the art, Information Fusion, 33, 100-112, 2017.

[4] Nirmala D.E., Vaidehi V., Comparison of Pixel-level and feature level image fusion methods, Proc. of the $2^{\text {nd }}$ International Conference on Computing for Sustainable Global Development (INDIACom), New Delhi, 743-748, 2015. 
[5] Burt P., Adelson E., The Laplacian Pyramid as a compact image code, IEEE Transactions on Communications, 31(4), 532-540, 1983.

[6] Zapłotny M., Damage identification in aircraft composite structures using information fusion methods, Master Thesis, Silesian University of Technology, Faculty of Mechanical Engineering, Gliwice, 2017.

[7] Katunin A., Kostka P., Characterisation of impact damage of composite structures using wavelet-based fusion of ultrasonic and optical images, Advanced Composites Letters 23(5), 123-130, 2014.

[8] Yang B., Li S., Multifocus image fusion and restoration with sparse representation, IEEE Transactions on Instrumentation and Measurement, 59(4), 884-892, 2010.

[9] Al-Wassai F.A., Kalyankar N.V., Al-Zuky A.A., The IHS transformations based image fusion, arXiv:1107.4396, 2011.

[10] LLC Black Ice Software. HSI color space - color space conversion. www.blackice. com, accessed at 11.07.2017.

[11] Choi M., A new Intensity-Hue-Saturation fusion approach to image fusion with a tradeoff parameter, IEEE Transactions on Geoscience and Remote Sensing, 44(6), 1672-1682, 2006.

[12] Amolins K., Zhang Y., Dare P., Wavelet based image fusion techniques - An introduction, review and comparison, ISPRS Journal of Photogrammetry and Remote Sensing, 62(4), 249-263, 2007.

[13] Piella G., New quality measure for image fusion. Proc. of the $7^{\text {th }}$ International Conference on Information Fusion, Stockholm, 2004.

[14] Wang Z., Borovick A.C., A universal image quality index, IEEE Signal Processing Letters, 9, 81-84, 2002. 